\title{
Estudo das características epidemiológicas e clínicas de 332 casos de leishmaniose tegumentar notificados na região norte do Estado do Paraná de 1993 a 1998
}

\author{
Epidemiological and clinical study of 332 cases of cutaneous leishmaniasis \\ in the north of Parana State from 1993 to 1998
}

Edilene Alcântara de Castro', Vanete Thomaz Soccol'1, Norberto Membrive² e Ennio Luz'

\begin{abstract}
Resumo Casos de leishmaniose tegumentar no norte do Estado do Paraná têm sido assinalados desde os anos 50, mas no final dos anos 80, houve um surto epidêmico. Para conhecer o perfil epidemiológico da leishmaniose cutânea na região Norte do Paraná foi feito um levantamento de casos, entre 1993 e 1998. Foram notificados 316 casos da doença em 35 municípios paranaenses. Dezesseis casos eram importados de outros estados e do Paraguai. Os indivíduos do sexo masculino (61,2\%), na faixa etária de 15 a 49 anos (70,8\%) representaram a maioria dos casos de leishmaniose tegumentar. Sessenta e sete porcento dos pacientes apresentaram lesões únicas, 31\% lesões múltiplas e 2\% lesões de mucosas. Num total de 367 lesões analisadas 47,7\% estavam localizadas nos membros inferiores, 26,7\% nos membros superiores e $16 \%$ na face. Trinta e duas cepas de Leishmania isoladas foram identificadas, com base em 13 isoenzimas, como pertencentes à espécie Leishmania (Viannia) braziliensis.
\end{abstract}

Palavras-chaves: Epidemiologia. Leishmaniose tegumentar. Estado do Paraná. Brasil. Leishmaniose humana.

Abstract Cases of cutaneous leishmaniasis (CL) in northern Parana State have been reported since the 1950's, but towards the end of the 1980's there has been an increase in the number of human infections. From 1993 to 1998, a study was carried out in an attempt to define the epidemiological profile of leishmaniasis in this region. A total of 316 cases of $C L$ were reported from 35 municipal districts and 16 imported cases. Most cases of $C L$ notified (70.8\%) were in the age group of 15 to 49 years old and most of these were males (61.2\%). Of the 332 positive patients, $66.8 \%$ presented single classic lesions and $31.1 \%$ multiple lesions. Most of these lesions were located in the lower limbs (47.7\%) while $26.7 \%$ were in the arms and $16 \%$ on the face. Thirty-two Leishmania stocks were isolated and identified by isoenzymatic characterization using 13 enzymatic systems as Leishmania (Viannia) braziliensis.

Key-words: Epidemiology. Cutaneous leishmaniasis. Paraná State. Brazil human leishmaniasis.

A leishmaniose é uma zoonose causada por protozoário parasita do gênero Leishmania, cujo ciclo tem a participação de um hospedeiro vertebrado e um invertebrado. Durante seu ciclo biológico, este protozoário apresenta duas formas: amastigota e promastigota. A forma amastigota parasita o fagossoma das células do SFM (Sistema Fagocítico Mononuclear) dos hospedeiros vertebrados (homem, animais selvagens e animais domésticos). A forma promastigota habita o aparelho digestivo do vetor, insetos da família Psychodidae, subfamília Phlebotominae.
A leishmaniose tem distribuição mundial, com um número estimado de 350 milhões de pessoas vivendo em regiões endêmicas correndo o risco de contrair a infecção ${ }^{15}$. O gênero Leishmania, no Novo Mundo, ocorre desde a península de Yucatã, no México, até o norte da Argentina, por onde distribuem-se pelo menos 17 taxa, causando diversas formas clínicoepidemiológicas. Destes, dez taxa são assinalados parasitando o homem ${ }^{22}$. No Brasil sete taxa circulam: Leishmania (Leishmania) amazonensis, nas regiões norte e nordeste, Leishmania (Viannia) guyanensis, na

\footnotetext{
1. Laboratório de Parasitologia Molecular do Departamento de Patologia Básica do Setor de Ciências Biológicas da Universidade Federal do Paraná. 2. Laboratório de Entomologia da Fundação Nacional de Saúde, Regional de Arapongas, PR.

Trabalho parcialmente financiado pelo CNPq.

Endereço para correspondência: Dra. Vanete Thomaz Soccol. Centro Politécnico/UFPR, Jardim das Américas, 81531-990 Curitiba, PR.

Tel: 41 361-1701, Fax: 41 266- 2042

e-mail:vasoccol@bio.ufpr.br

Recebido para publicação em 17/4/2001.
} 
região norte, Leishmania (Viannia) braziliensis da região norte até o sul, e Leishmania (Viannia) shawi, Leishmania (Viannia) naiffi e Leishmania (Viannia) lainsoni na região norte, e, finalmente Leishmania (Leishmania) infantum no norte, nordeste e parte do sudeste do país ${ }^{21015}$. A leishmaniose tegumentar ocorre em quase todos os estados e acomete pessoas de todas as faixas etárias e de ambos os sexos ${ }^{13}$. No Brasil, entre os anos de 1956 e 1976 foram registrados 33.991 casos em humanos. Nos últimos 15 anos (1985-1999), foram registrados no país 388.155 casos autóctones de leishmaniose tegumentar?.

A leishmaniose tegumentar está presente em duas áreas geográficas distintas do Paraná apresentando comportamento epidemiológico diferente. Em uma das áreas, localizada no Vale do Rio Ribeira, a doença é conhecida desde o começo do século. Na outra, no norte do Paraná, têm sido assinalados casos de LT desde o início de sua colonização. Após a campanha de controle da malária, significativa redução do número de casos foi observada até o final dos anos 80 ( $E$. Luz: informação pessoal). No final dos anos 80 e inicio dos anos 90 , houve um aumento do número de pacientes, atingindo cifras na ordem de 1359 em $1994^{6}$. Silveira et a/19 20 , realizaram trabalhos no noroeste do Paraná assinalando a presença de Leishmania (Viannia) braziliensis e Leishmania (Leishmania) amazonensis.

$\mathrm{Na}$ região norte do Estado, a espécie de flebotomíneo prevalente é Lutzomyia whitmani ${ }^{1121}$. Luz et $\mathrm{al}^{11}$ encontraram nesta região fêmeas de Lutzomyia whitmani infectadas por Leishmania (Viannia) braziliensis confirmando o papel vetorial da espécie.

No presente trabalho é feito um estudo retrospectivo e descritivo da leishmaniose tegumentar na região norte do Paraná. O objetivo é avaliar a condição de risco para a ocorrência de leishmaniose tegumentar e evidenciar o padrão epidemiológico da infecção em humanos.

\section{MATERIAL E MÉTODOS}

Área de Estudo. O Estado do Paraná, pertence ao Planalto Meridional e Região Sul do Brasil, localizado entre $22^{\circ} 29^{\prime} 23^{\prime \prime}$ a $26^{\circ} 42^{\prime} 59^{\prime \prime}$ latitude sul e 4802'24" a 5437'38" longitude oeste. Com $201.000 \mathrm{~km}^{2}$, ocupa posição de transição entre tropicalidade e subtropicalidade . Cerca de um quarto do seu território fica na Zona Equatorial (ao norte da linha de Capricórnio) e o restante na Zona Temperada do Sul. Tal localização determina como uma área de contatos e transição em termos físicos e naturais, apresentando diversas ocorrências de clima, solo e cobertura vegetal, bem como uma diferenciada formação geológica e conformação geomorfológica1.

A área de estudo é definida climaticamente como $\mathrm{Cfa}: \mathrm{C}=$ média do mês mais frio inferior a $18^{\circ} \mathrm{C} ; \mathrm{f}=\mathrm{sem}$ estação seca definida e; $a=$ verões quentes. $A$ precipitação pluviométrica anual é de $2.000 \mathrm{~mm}$ que cai em aproximadamente 120 dias. Apesar da chuva se distribuir durante o ano todo (clima subtropical), verificase maior concentração nos meses de novembro e janeiro. As temperaturas registrando médias de 20 a $22^{\circ} \mathrm{C}$, enquanto a média das máximas acusa 30 a $32^{\circ} \mathrm{C}$ e as máximas absolutas $38^{\circ} \mathrm{C}$. Em meados do ano (inverno) as médias das mínimas varia entre 10 e $12^{\circ} \mathrm{C}$ e as mínimas absolutas acusam $2^{\circ} \mathrm{C}$ negativos, com a ocorrência de 3 a 5 dias de geada por ano. As altitudes são de aproximadamente $650 \mathrm{~m}$. Os teores de umidade variam entre 75 e $95 \%{ }^{25}$.

A ocupação do solo, especialmente intensa nesta porção do estado, fez com que da cobertura vegetal originária restassem apenas algumas áreas testemunhas (ilhotas residuais) ou associada à presença de cursos de água (floresta ciliares). A colonização, as estradas rodoviárias e ferroviárias, mas, em especial, a atividade agrícola, foram os responsáveis pela transformação acelerada da paisagem. Esta região foi colonizada nos anos 30 e 40, com a expansão da cultura cafeeira, destruindo a floresta original para dar lugar aos pés de café. Hoje a população da área rural representa, em alguns municípios, menos de $20 \%$ da população total. A densidade demográfica nos municípios mais populosos atinge 202,3 habitantes $/ \mathrm{km}^{2}$, sendo que a maioria reside em zona urbana (Figura 1).

Coleta de dados epidemiológicos. Nas buscas ativas todos os pacientes encontrados com lesões eram encaminhados a Regional de Arapongas - FUNASA para diagnóstico. Os demais pacientes foram encaminhados por dermatologistas ou agentes de saúde desta ou de outras regionais. Um dos autores (Membrive N, FUNASA) era o responsável pela coleta de dados e preenchimento das fichas de notificação e posterior encaminhamento à Secretaria de Estado de Saúde do Paraná. Nestas fichas são registrados dados dos pacientes como profissão, idade, sexo, número e local de lesões, tempo e local provável da infecção, diagnóstico e tratamento.

Diagnóstico. Os casos de leishmaniose tegumentar assinalados nos municípios estudados foram diagnosticados pelo teste de Intradermorreação de Montenegro e confirmados por exame parasitológico de material da lesão pelos seguintes métodos: in print, biópsia ou meio de cultivo. Cem porcento das lesões foram submetidas ao exame de Intradermorreação e in print.

O teste de Montenegro foi realizado, empregando-se antígeno de Leishmania fornecido pelo CPPI - Secretaria de Estado de Saúde do Paraná. Foram consideradas positivas as reações cujo diâmetro da pápula foi igual ou superior a 5mm, após 48 a 72 horas da inoculação.

Trinta e quatro pacientes com intradermorreação positiva foram submetidos a biópsia da lesão e isolamento do parasito em meio de cultivo (NNN e Tobbie \& Evans). As cepas isoladas foram identificadas por isoenzimas segundo Thomaz-Soccol ${ }^{22}$. 


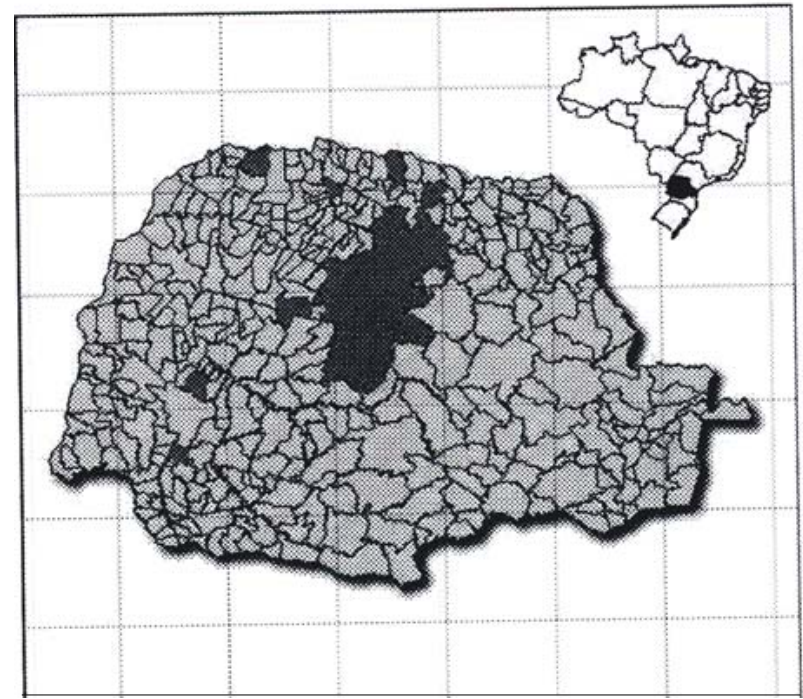

Figura 1 - Distribuição geográfica dos 35 municípios do Estado do Paraná com casos de leishmaniose tegumentar notificados em humanos, nos anos de 1993 - 1998, atendidos pela Regional de Arapongas - FUNASA.

\section{RESULTADOS}

Na região em estudo, no período entre 1993 e 1998, 332 novos casos de leishmaniose tegumentar foram notificados, com maior número em 1993 e 1994 e menor número em 1998. Deste total, 316 pacientes eram provenientes de 35 municípios do estado do Paraná, 15 eram casos importados dos estados do Mato Grosso, Rondônia e Amazonas e um do Paraguai. Dos 35 municípios do Paraná os que apresentaram maior número de casos de leishmaniose tegumentar foram Apucarana (91), Arapongas (41), Kaloré (19), Cambira (19) e Borrazópolis (17) (Tabela 1). 198 casos (59,6\%) eram autóctones da localidade onde os pacientes residiam e $67(20,2 \%)$ eram autóctones do município, ou seja, os pacientes relataram ter se infectado em atividades de lazer ou trabalho em localidades pertencentes ao mesmo município.

Os indivíduos do sexo masculino representaram $61,2 \%$ dos casos de LT notificados e a faixa etária de 15 a 49 anos foi a mais atingida com 70,8\% (Figura 2). Quanto à ocupação profissional, casos de LT foram assinalados entre as categorias de lavradores $(38 \%)$, mulheres do lar (22\%), estudantes (15\%) e outros $(15 \%)$.

Durante o período de estudo, um total de 410 pacientes com lesões cutâneas, foram submetidos ao teste de intradermorreação. Destes, 332 apresentaram reação cutânea compatível com leishmaniose que foi, posteriormente, confirmada por exame parasitológico.
Num total de 367 lesões assinaladas, $67 \%$ eram lesões únicas e $31 \%$ eram lesões múltiplas (2 ou mais). Apenas $2 \%$ corresponderam a leishmaniose cutâneo-mucosa. As lesões cutâneas estavam localizadas preferencialmente nos membros inferiores $(47,7 \%)$, seguidos dos membros superiores $(26,7 \%)$ e face (16\%) (Figura 3).

A forma clínica mais comumente encontrada foi, em $99 \%$ dos casos, a lesão ulcerativa, sendo as restantes do tipo nodular. Em $70,8 \%$ dos casos, o tempo médio de evolução da lesão foi de 4 a $12 \pm 2$ semanas segundo relato dos pacientes atendidos. Nos meses de junho a setembro foram assinalados o maior número de casos, totalizando $51,8 \%$ (Figura 4).

O coeficiente médio de detecção dos casos de leishmaniose tegumentar por 100.000 habitantes foi de 4,81, para os anos de 1993 a 1998, em 35 municípios do estado do Paraná. Os coeficientes de detecção com valores baixos (entre 0 e 3 ) foram assinalados em doze municípios. Outros onze municípios apresentaram coeficiente de detecção com valores médios entre 3 e 11. Em doze municípios, os coeficientes foram altos (entre 11 e 70) e variaram entre 11,01 (Jandaia do Sul) e 62,78 (Kaloré) (Tabela 1).

Trinta e duas cepas de Leishmania foram isoladas sendo 31 de lesões cutâneas e 1 de mucosas. As cepas isoladas foram identificadas com base em 13 isoenzimas como pertencentes à espécie Leishmania (Viannia) braziliensis ${ }^{23}$.

\section{DISCUSSÃO}

Desde a descoberta do gênero Leishmania por Ross, em $1903^{18}$, até o presente, houve uma mudança no aspecto epidemiológico da leishmaniose. Antes, a leishmaniose cutânea era considerada, basicamente, uma zoonose de animais selvagens, sendo transmitida acidentalmente ao homem quando este adentrava nas 
florestas para exploração, extração de madeira, abertura de estradas e mineração. A leishmaniose comportava-se, portanto, como doença profissional, acometendo apenas adultos do sexo masculino. Este padrão epidemiológico também foi observado no início da colonização do Estado do Paraná (entre 1932 e $1955)^{12}$. Neste período foram registrados 2.902 casos de leishmaniose cutânea associados à derrubada das matas para o plantio de café e com $89,9 \%$ dos casos assinalados em homens adultos.

Na região norte do Estado do Paraná, a colonização teve início na década de 20 e alcançou seu apogeu nas décadas de 30 e $40 \mathrm{com}$ a substituição da floresta original por plantações de café. Naquela época a leishmaniose não era doença de declaração obrigatória e por isto é difícil estimar o número de casos anuais ocorridos. Um dos poucos registros encontrados é o de Miranda et al $^{12}$ que relatam os primeiros casos no Paraná registrados em 1918 (Guaíra) e 1920 (Jacarezinho). Acreditavam os autores que a introdução da leishmaniose no Paraná teria tido duas origens: uma oriunda do Mato Grosso e Paraguai e outra de São Paulo e uma pandemia teria ocorrido entre 1920 e $1944^{12}$.

Este caráter, tipicamente silvestre, permanece ainda hoje em regiões do norte e centro-oeste do Brasil, regiões que somente agora estão sendo colonizadas e com um intenso processo de exploração ${ }^{10}$.

Em algumas regiões do país intensas modificações do ambiente ocorreram, devido a ação do homem, em sua atividade agrícola e pastoril, o que levou ao quase desaparecimento da leishmaniose cutânea no final da década de 40. Todavia, a partir das década de 70 e 80

Tabela 1 - Distribuição dos casos de leishmaniose tegumentar em humanos, nos anos de 1993 - 1998 , nos municípios atendidos pela Regional de Arapongas - FUNASA, Estado do Paraná, Brasil.

\begin{tabular}{|c|c|c|c|c|c|c|c|c|}
\hline Municípios & 1993 & 1994 & 1995 & 1996 & 1997 & 1998 & Total & $C D^{*}$ \\
\hline Apucarana & 27 & 36 & 14 & 4 & 6 & 4 & 91 & 14,06 \\
\hline Arapongas & 19 & 11 & 2 & 7 & 2 & 0 & 41 & 7,99 \\
\hline Kaloré & 6 & 7 & 1 & 3 & 1 & 1 & 19 & 62,78 \\
\hline Cambira & 3 & 3 & 6 & 4 & 2 & 1 & 19 & 47,34 \\
\hline Borrazópolis & 4 & 9 & 0 & 0 & 4 & 0 & 17 & 29,97 \\
\hline Bom Sucesso & 6 & 6 & 3 & 0 & 0 & 0 & 15 & 40,50 \\
\hline Nova Tebas & 0 & 0 & 6 & 4 & 3 & 0 & 13 & 22,85 \\
\hline Jandaia do Sul & 5 & 4 & 4 & 0 & 0 & 0 & 13 & 11,01 \\
\hline Grandes Rios & 0 & 5 & 2 & 0 & 0 & 3 & 10 & 21,18 \\
\hline Rolandia & 4 & 0 & 2 & 0 & 0 & 0 & 6 & 2,02 \\
\hline Londrina & 1 & 0 & 0 & 3 & 1 & 0 & 5 & 0,18 \\
\hline Faxinal & 2 & 0 & 2 & 1 & 0 & 0 & 5 & 5,33 \\
\hline São Jorge do Ivaí & 1 & 0 & 4 & 0 & 0 & 0 & 5 & 14,90 \\
\hline Marumbi & 0 & 2 & 0 & 0 & 1 & 2 & 5 & 18,06 \\
\hline Ivaiporã & 5 & 0 & 0 & 0 & 0 & 0 & 5 & 2,58 \\
\hline Corbélia & 4 & 1 & 0 & 0 & 0 & 0 & 5 & 5,27 \\
\hline Mauá da Serra & 1 & 0 & 0 & 2 & 1 & 0 & 4 & 10,30 \\
\hline Pitanga & 0 & 0 & 3 & 1 & 0 & 0 & 4 & 1,85 \\
\hline Novo Itacolomi & 0 & 0 & 1 & 2 & 1 & 0 & 4 & 23,26 \\
\hline Rio Bom & 0 & 3 & 1 & 0 & 0 & 0 & 4 & 18,78 \\
\hline California & 0 & 2 & 1 & 1 & 0 & 0 & 4 & 8,68 \\
\hline Marilândia & 0 & 1 & 1 & 1 & 0 & 0 & 3 & 5,51 \\
\hline Sabáudia & 0 & 0 & 0 & 2 & 1 & 0 & 3 & 9,23 \\
\hline Porecatu & 3 & 0 & 0 & 0 & 0 & 0 & 3 & 3,14 \\
\hline Florestópolis & 0 & 0 & 0 & 2 & 0 & 0 & 2 & 2,73 \\
\hline Itambé & 1 & 1 & 0 & 0 & 0 & 0 & 2 & 5,60 \\
\hline Sertanópolis & 0 & 0 & 0 & 1 & 0 & 0 & 1 & 1,10 \\
\hline São Pedro do Ivaí & 0 & 0 & 0 & 0 & 1 & 0 & 1 & 1,73 \\
\hline Manoel Ribas & 1 & 0 & 0 & 0 & 0 & 0 & 1 & 1,27 \\
\hline Godoy Moreira & 0 & 1 & 0 & 0 & 0 & 0 & 1 & 4,34 \\
\hline Lobato & 0 & 0 & 1 & 0 & 0 & 0 & 1 & 4,10 \\
\hline Terra Rica & 0 & 0 & 1 & 0 & 0 & 0 & 1 & 1,20 \\
\hline C. Leonidas Marques & 0 & 1 & 0 & 0 & 0 & 0 & 1 & 1,15 \\
\hline Lunardelli & 1 & 0 & 0 & 0 & 0 & 0 & 1 & 1,06 \\
\hline Campo Mourão & 1 & 0 & 0 & 0 & 0 & 0 & 1 & 0,20 \\
\hline Total & 95 & 93 & 55 & 38 & 24 & 11 & 316 & 4,81 \\
\hline
\end{tabular}

$C D^{*}$ - Coeficiente de deteç̧ão de casos de leishmaniose tegmentar por 100.000 habitantes. 


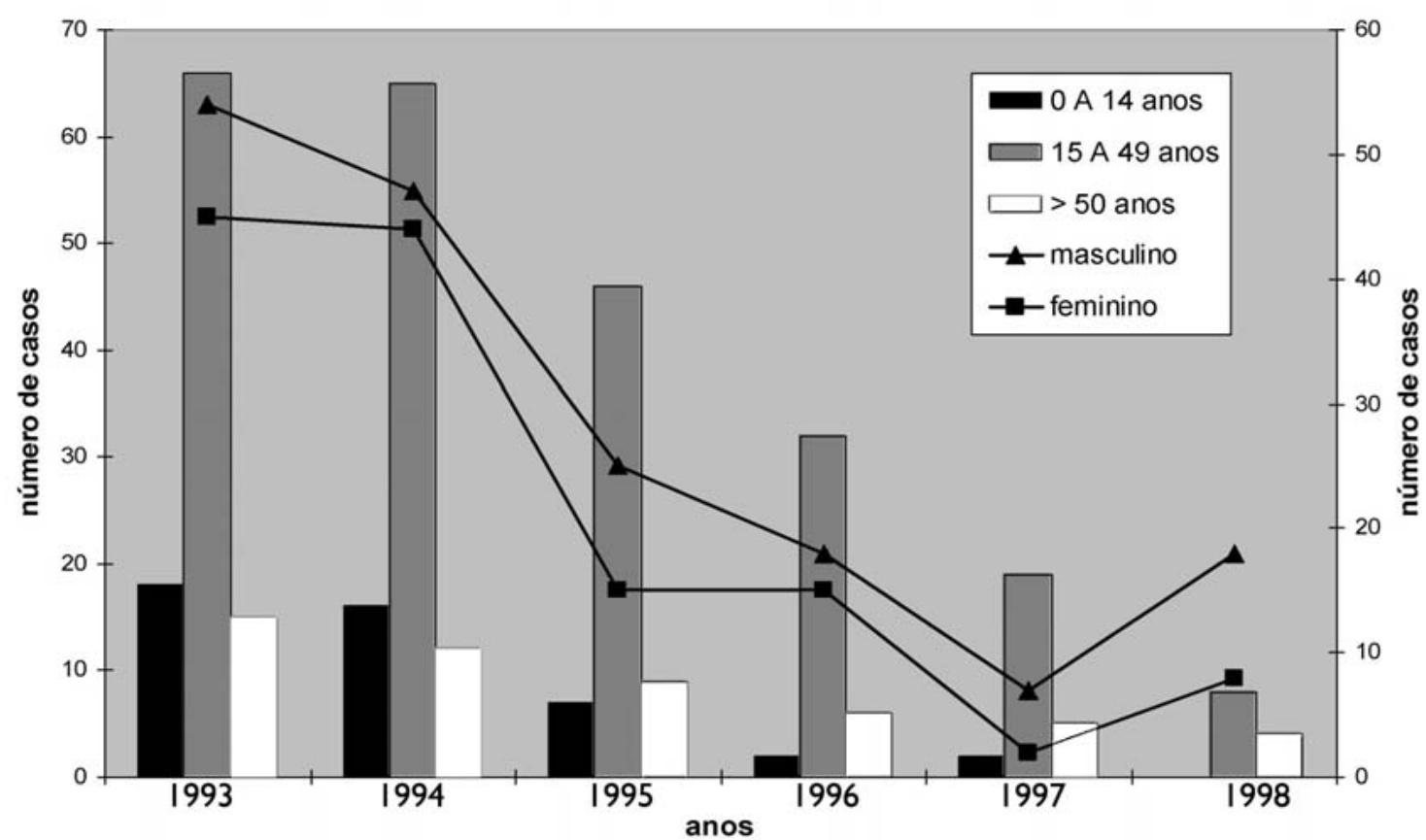

Figura 2 - Distribuicão por faixa etária e sexo dos casos de leishmaniose tegumentar em humanos, nos anos de 1993 - 1998, notificados nos 35 municípios do Estado do Paraná, atendidos pela Regional de Arapongas FUNASA.

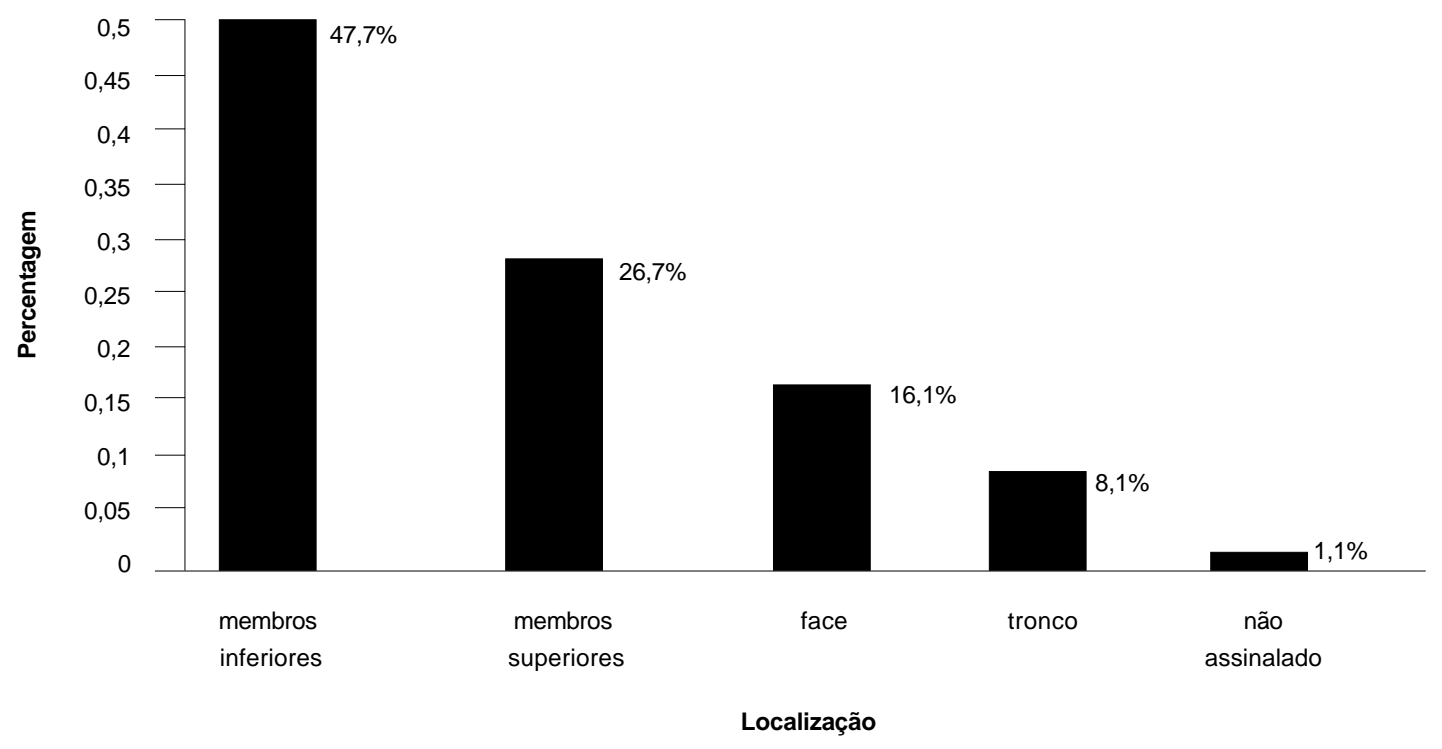

Figura 3 - Distribuição anatômica das lesões encontradas nos pacientes de leishmaniose tegumentar atendidos pela Regional de Arapongas - FUNASA, nos anos de 1993 - 1998, Estado do Paraná. 


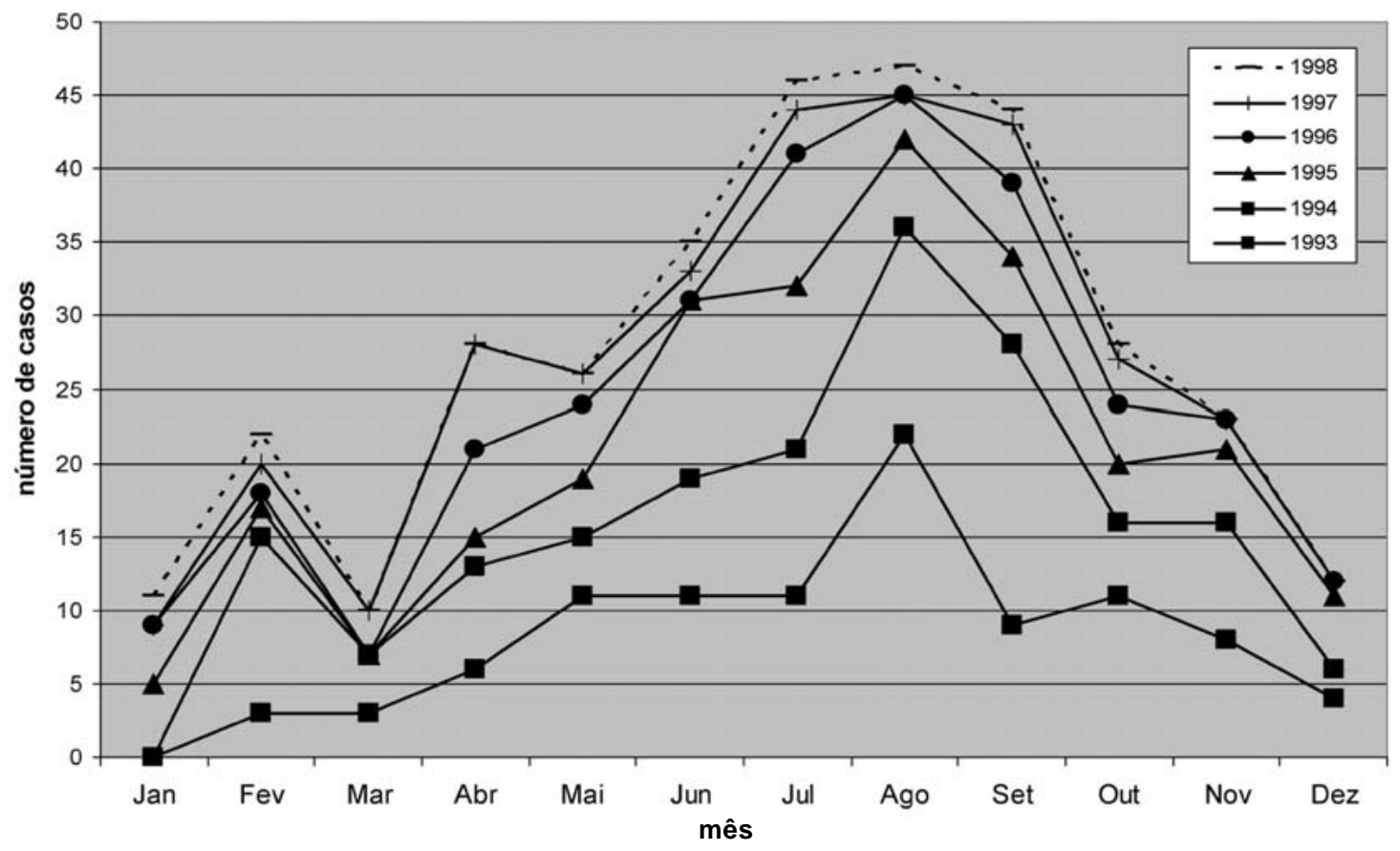

Figura 4 - Distribuição mensal dos casos de leishmaniose tegumentar nos anos de 1993 - 1998, notificados na Regional de Arapongas - FUNASA, Estado do Paraná, Brasil.

a leishmaniose reaparece, nestas regiões, com aumento significativo no número de casos e com o surgimento de novas áreas endêmicas 345817192026 .

O perfil da leishmaniose no Estado do Paraná não diferiu daquele assinalado para as outras regiões do Brasil. $\mathrm{Na}$ década de 90, apenas resíduos de mata permanecem nas áreas ciliares e refúgios esporádicos entre plantações. As características eco-geográficas destas pequenas ilhas de matas favorecem 0 aparecimento de pequenos focos de leishmaniose, pois há umidade necessária ao desenvolvimento dos flebotomíneos vetores. Outro fator interessante observado é que as casas, nas zonas rurais, são construídas ao lado destes refúgios, não distanciando 150 a 300 metros da mata. E, entre a reserva florestal e as residências, existem plantas frutíferas, bambusais e outras vegetações arbustivas que permitem a passagem dos flebotomíneos da reserva florestal ao peridomicílio (Figura 5). Portanto, os indivíduos que trabalham na agricultura ou que têm atividade de lazer, em contato com estas reservas florestais, não estando imunizados, correm o risco de contrair leishmaniose na referida região. Embora estudantes de pouca idade e mulheres com atividades domésticas representem alta porcentagem dos casos de LT nestas regiões, isto, provavelmente não seja devido à infecção intradomiciliar. Pois, as crianças, mesmo pequenas, e mulheres acompanham os homens na lavoura. Muitos meninos, a partir de oito anos ou menos, realizam atividades

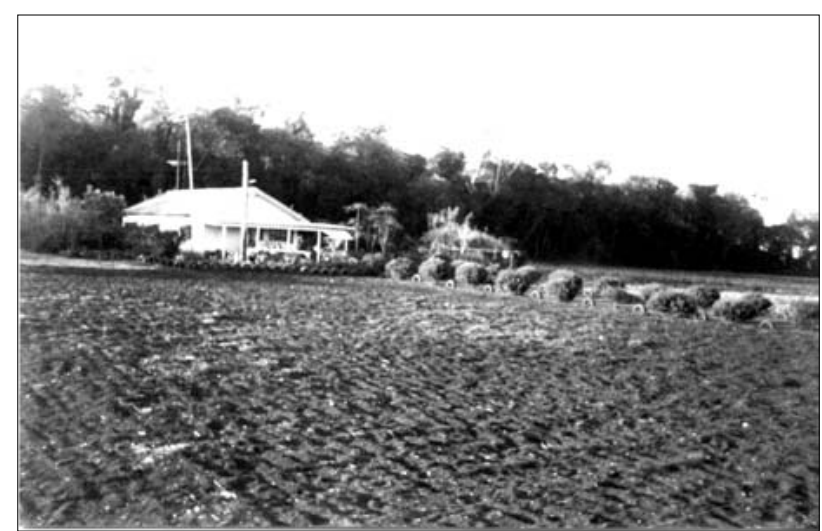

Figura 5 - Residência localizada na zona rural da região norte do Estado do Paraná, Brasil.

extradomiciliares, penetrando na mata adjacente às residências para procurar lenha, caçar, pescar ou outras atividades de lazer, podendo haver transmissão peridomiciliar ou na própria mata.

Neste trabalho, o perfil etário da população infectada está representado pela faixa de 15 a 49 anos (70,8\%) com predomínio de indivíduos do sexo masculino $(61,2 \%)$ sendo $38 \%$ lavradores, embora tenham sido observados casos em crianças. Silveira et al, 1999'19, realizaram trabalhos no noroeste do Paraná assinalando $68,9 \%$ dos pacientes na faixa etária de 15 a 49 anos e $54,8 \%$ eram do sexo masculino, percentuais muito 
semelhantes aos encontrado neste trabalho. Outros autores, nas regiões nordeste e sudeste do país, também relataram a ocorrência do mesmo perfil epidemiológico ${ }^{913141624}$.

O coeficiente médio de detecção de casos de leishmaniose tegumentar por 100.000 habitantes encontrado para a região foi de 4,81 . Este valor representa a média de seis anos de estudo. No ano de 1994, onde foi observado o maior número de casos, este coeficiente chegou a 8,51. Todavia, estes valores foram sempre inferiores aos assinalados para outras regiões do Brasil ${ }^{7}$. Portanto, a leishmaniose tegumentar no Estado do Paraná caracteriza-se como endêmica. Como área de endemismo observa-se que as condições em que ocorre a transmissão permanecem estáveis, sob condições normais, durante muito tempo (anos). Em casos de alteração destas condições, em uma localidade específica, como mudanças extremas de clima, novos casos surgem em maior proporção na população atingindo as faixas etárias de indivíduos mais jovens.

\section{AGRADECIMENTOS}

Os autores expressam seus agradecimentos à Fundação Nacional da Saúde, Regional do Estado do Paraná.

\section{REFERÊNCIAS BIBLIOGRÁFICAS}

1. Camargo JB. Geografia Fisica, Humana e Econômica. 2' edição, 1998.

2. Dedet JP, Pradinaud R, Gay F. Epidemiological aspects of human cutaneous leishmaniasis in French Guyana. Transactions of Royal Society of Tropical Medicine and Hygiene 83: 616620, 1989.

3. Forattini OP, Pattoli DBG, Serra OP, Rocha e Silva EO, Rabello EX. Nota sobre leishmaniose tegumentar no litoral sul do Estado de São Paulo, Brasil. Revista de Saúde Pública 7:447452, 1973.

4. Forattini OP, Rabello EX, Pattoli DBG, Ferreira OA. Nota sobre um foco de leishmaniose tegumentar na região nordeste do Estado de São Paulo, Brasil. Revista de Saúde Pública 6:103105, 1972.

5. Forattini OP, Rabello EX, Serra OP, Cotrim MG, Galati EA, Barata JM. Observações sobre a transmissão da leishmaniose tegumentar no Estado de São Paulo, Brasil. Revista de Saúde Pública 10:31-43, 1976.

6. Fundação Nacional de Saúde. Casos notificados de leishmaniose tegumentar americana. Residentes na UF, por ano, segundo Unidade Federada, Brasil - 1980 - 1994. Ministério da Saúde, Brasília, 1995.

7. Fundação Nacional de Saúde. Manual de Controle da Leishmaniose Tegumentar Americana. Ministério da Saúde, Brasília, DF, 2000.

8. Gomes AC, Neves VL. Estratégia e perspectivas de controle da leishmaniose tegumentar no Estado de São Paulo. Revista Sociedade Brasileira de Medicina Tropical 31:553-558, 1998.

9. Jones TC, Johnson WD, Barreto AC, Lago E, Badaro R, Cerf B. Epidemiology of American Cutaneous Leishmaniasis due to Leishmania braziliensis braziliensis. The Journal of Infectious Diseases 156:73-83, 1987.

10. Lainson R, Shaw JJ. Evolution, classification and geographical distribution. In: Peters W, Killick-Kendrick R (eds) The Leishmaniases in Biology and Medicine, Academic Press, London, volume 1, p. 1987.

11. Luz E, Membrive N, Castro EA, Dereure J, Pratlong F, Dedet JA, Pandey A, Thomaz-Soccol V. Lutzomyia whitmani (Diptera: Psychodidae) as vector of Leishmania (V.) braziliensis in Paraná state, southern Brazil. Annals of Tropical Medicine \& Parasitology 94:623-631, 2000.

12. Miranda RN, Cunha C, Schweidson J. A Leishmaniose Tegumentar no Paraná. Revista Médica do Paraná 24:1-21, 1955.

13. Nunes V, Dorval M, Oshiro E, Noguchi R, Arão L, Hans G, Espíndola M. Estudo epidemiológico sobre leishmaniose tegumentar (LT) no município de Corguinho, MS - Estudos na população humana. Revista da Sociedade Brasileira de Medicina Tropical 28:185-193, 1995.

14. Oliveira-Neto MP, Pirmez C, Rangel E, Schubach A, Grimaldi Jr G. An outbreak of American cutaneous leishmaniasis in a periurban area of Rio de Janeiro: clinical and epidemiological. Memórias do Instituto Oswaldo Cruz 83:427-435, 1988.

15. Organisation Mondiale de la Santé. Controle des Leishmanioses. OMS, Genebra, vol. 793, 1990.

16. Pereira CAC, Tristão OC, Barbosa MCB, Pimentel AFM, Carvalho MTF. Leishmaniose tegumentar americana: mapeamento de área endêmica. Anais Brasileiros de Dermatologia 70:254-256, 1995.

17. Pignatti MG, Mayo RC, Alves MJCP, Souza SSAL, Macedo F, Pereira RM. Leishmaniose tegumentar americana na região nordeste do Estado de São Paulo - Brasil. Revista da Sociedade Brasileira de Medicina Tropical 28:243-247, 1995.

18. Ross $\mathrm{E}$. Notes on the bodies recently described by Leishmann and Donovan. Britannic Medicine Journal 2:1261-1262, 1903.

19. Silveira TGV, Arraes SMAA, Bertolini DA, Teodoro U, Lonardoni MVC, Roberto ACBS. Observações sobre o diagnóstico laboratorial e a epidemiologia da leishmaniose tegumentar no Estado do Paraná, sul do Brasil. Revista da Sociedade Brasileira de Medicina Tropical 32:413-423, 1999.

20. Silveira TGV, Teodoro U, Lonardoni MVC, Guilherme ALF, Toledo MJO, Ramos M, Arraes SMAA, Bertolini DA, Spinoza RP, Barbosa OC. Aspectos epidemiológicos da leishmaniose tegumentar em área endêmica do Estado do Paraná, Brasil. Cadernos de Saúde Pública 12:141-147, 1996

21. Teodoro U, Kuhl JB, Thomaz-Soccol V, Barbosa OC, Ferreira MEMC, Lozovei AL, Silveira TGV Influence of peri-domiciliar environmental conditions in the dispersion and proliferation of phlebotomine sandflies in Paraná State, southern Brazil. Brazilian Archives of Biology and Technology 40:747-751, 1997.

22. Thomaz-Soccol V. Les Leishmania du Noveau Monde. Analyse enzymatique. Demarche progressive phenetique-cladistique. Tese, Doctorat, Université Montpellier I. Faculté de Medicine, Montpellier, France, 1993.

23. Thomaz-Soccol V, Castro EA, Luz E, Dereure J, Pratlong F, Dedet JA, Leishmania braziliensis: Biochemical Characterisation by Isoenzyme Electrophoresis in two regions of Paraná State, 
Brazil. In: Abstracts of International Conference on New Horizons in Biotechnology, Trivandrum, India, p. 225, 2001.

24. Tolezano JE. Ecoepidemiological aspects of American cutaneous leishmaniasis in the state of São Paulo, Brazil. Memórias do Instituto Oswaldo Cruz 89:427-434, 1994.
25. Troppmair H. Perfil Fitoecológico do Estado do Paraná. Boletim de G0,eografia da Universidade Estadual de Maringá 8: 67-81, 1990.

26. Verzignassi TGV. Leishmaniose tegumentar americana: aspectos epidemiológicos no Norte do Paraná - Brasil. Ciência e Cultura 40:884-885, 1988. 